EPJ Web of Conferences 63,02009 (2013)

DOI: 10.1051 /epjconf/ 20136302009

(C) Owned by the authors, published by EDP Sciences, 2013

\title{
Brownian shape dynamics in fission
}

\author{
Jørgen Randrup ${ }^{1, a}$ and Peter Möller ${ }^{2}$ \\ ${ }^{1}$ LBNL, Berkeley, California 94720, USA \\ ${ }^{2}$ LANL, Los Alamos, New Mexico, 87545, USA
}

\begin{abstract}
It was recently shown that remarkably accurate fission-fragment mass distributions are obtained by treating the nuclear shape evolution as a Brownian walk on previously calculated five-dimensional potentialenergy surfaces; the current status of this novel method is described here.
\end{abstract}

\section{Introduction}

As discussed already in the pioneering works by Meitner and Frisch [1] and Bohr and Wheeler [2, 3] in 1939, nuclear fission can be viewed qualitatively as an evolution of the nuclear shape from that of a single compound nucleus to two receding fragments. The character of the shape dynamics is still not well established but, as a step forward from a purely statistical approach toward a full dynamical treatment, it is interesting to explore scenarios in which the evolution is strongly dissipative $[4,5]$.

We therefore picture the nuclear fission process as an evolution of the nuclear shape from a relatively compact mononucleus to a dinuclear configuration. Generally, the shape is described by a set of parameters, $\chi=\left\{\chi_{i}\right\}$, whose time development is the result of a complicated interplay between a variety of effects. At the present stage, the shape is composed of three smoothly joined quadratic surfaces $[6,7]$, as illustrated in Fig. 1, and is thus described by five shape parameters.

Most basic is the potential energy associated with a given shape, $U(\chi)$, for which a number of relatively mature models have been developed. We employ potential-energy surfaces that have been calculated with the macroscopic-microscopic method in which the potential energy is the sum of shape-dependent macroscopic (liquid-drop type) terms and a microscopic correction that reflects the structure of the single-particle levels in the effective potential associated with the specified nuclear shape [8, 9],

$$
U(\chi)=U_{\text {macro }}(\chi)+U_{\text {micro }}(\chi)
$$

Such potential energies have been calculated for 5,254 nuclei with $171 \leq A \leq 330$ and tabulated on a fivedimensional lattice containing over five million different shapes. The potential energy provides the driving force, $\boldsymbol{F}^{\mathrm{pot}}(\chi)$, which has the components $F_{i}^{\mathrm{pot}}=-\partial U / \partial \chi_{i}$.

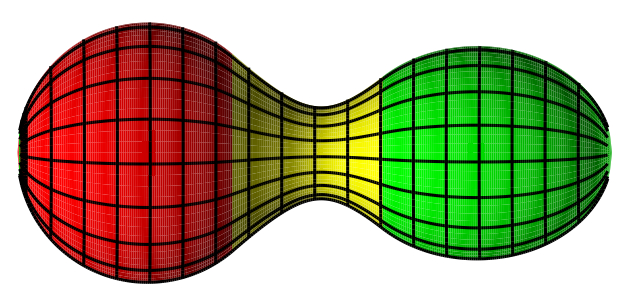

Figure 1. Illustration of the $3 \mathrm{QS}$ shape family employed: two spheroids are joined smoothly by a hyperboloid.

The driving force seeks to change the nuclear shape towards lower energies and the associated matter rearrangement gives rise to a collective kinetic energy, $\frac{1}{2} \dot{\boldsymbol{\chi}} \cdot \boldsymbol{M} \cdot \dot{\boldsymbol{\chi}}$. Furthermore, the shape degrees of freedom are coupled dissipatively to the internal degrees of freedom in the nuclear many-body system. We are assuming that the damping is strong. The resulting motion is then slow and, consequently, we neglect the acceleration terms, $\left\{\sum_{j} M_{i j} \ddot{\chi}_{j}\right\}$, as well as terms of second order in the velocities $\left\{\dot{\chi}_{i}\right\}$. The inertial mass tensor $\{\boldsymbol{M}(\boldsymbol{\chi})\}$ then plays no role in the shape evolution and it shall henceforth be neglected.

The dissipative coupling renders the shape evolution both damped and diffusive and it must therefore be treated by transport methods that allow for the stochastic elements of the dynamics. The dynamics is then akin to Brownian motion: The Brownian particle is the (five-dimensional) nuclear shape, while the fluid into which it is immersed is represented by the internal (nucleonic) degrees of freedom to which it is coupled.

\section{Brownian shape motion}

The average dissipative force provides a friction of the standard form, $\left\langle\boldsymbol{F}^{\text {diss }}\right\rangle=\boldsymbol{F}^{\text {fric }}=-\boldsymbol{\gamma} \cdot \boldsymbol{F}$, and we assume that the random remainder, $\boldsymbol{F}^{\mathrm{ran}}(t)$, is Markovian,

$$
\left\langle F_{i}^{\mathrm{ran}}(t) F_{j}^{\mathrm{ran}}\left(t^{\prime}\right)\right\rangle=2 T \gamma_{i j} \delta\left(t-t^{\prime}\right),
$$


where $T$ is the temperature of the nucleus when it has attained the shape $\chi$. The dissipation tensor $\gamma$ is positive definite and so is its inverse, the mobility tensor $\boldsymbol{\mu} \equiv \boldsymbol{\gamma}^{-1}$, which can be conveniently expressed in terms of its eigenvectors $\tilde{\chi}^{(n)}$, i.e. $\mu_{i j}=\sum_{n} \tilde{\chi}_{i}^{(n)} \tilde{\chi}_{j}^{(n)}$ and

$$
\boldsymbol{\mu} \cdot \tilde{\chi}^{(n)}=\mu^{(n)} \tilde{\chi}^{(n)}, \quad \sum_{i} \tilde{\chi}_{i}^{(n)} \cdot \tilde{\chi}_{i}^{\left(n^{\prime}\right)}=\mu^{(n)} \delta_{n n^{\prime}} .
$$

The shape evolution $\chi(t)$ is governed by the Smoluchowski equation of motion which balances the driving force from the potential against the full dissipative force: $\boldsymbol{F}^{\text {pot }}+\boldsymbol{F}^{\text {fric }}+\boldsymbol{F}^{\text {ran }} \doteq \mathbf{0}$. This condition immediately yields the instantaneous velocity at a given shape $\chi$,

$$
\dot{\chi}(t) \doteq \boldsymbol{\mu} \cdot\left[\boldsymbol{F}^{\mathrm{pot}}(\chi)+\boldsymbol{F}^{\mathrm{ran}}(\chi, t)\right] .
$$

This equation of motion can be simulated numerically by evaluating the shape change accumulated during a small time interval $\Delta t$,

$$
\delta \chi=\sum_{n} \tilde{\chi}^{(n)}\left[\Delta t \tilde{\chi}^{(n)} \cdot \boldsymbol{F}^{\mathrm{pot}}+\sqrt{2 T \Delta t} \xi_{n}\right]
$$

where the five random numbers $\left\{\xi_{n}\right\}$ are sampled from a distribution that has zero mean and unit variance (e.g. a normal distribution). The shape change $\delta \chi$ consists of an average displacement proportional to (but generally not aligned with) the driving force $\boldsymbol{F}^{\mathrm{pot}}(\boldsymbol{\chi})$ and a stochastic term arising from the random force $\boldsymbol{F}^{\mathrm{ran}}(\boldsymbol{\chi})$. On the basis of this expression, it is straightforward to solve the Smoluchowski equation by direct numerical simulation.

This type of evolution is exactly characteristic of Brownian motion. But it differs from the familiar text book example in several respects: 1) The motion occurs in five dimensions rather than three (the shape paramter $\chi$ is five-dimensional); 2) the medium is both non-uniform and anisotropic (the dissipation tensor $\gamma$ depends on $\chi$ and is generally not isotropic); and 3) there is an external driving force, $\boldsymbol{F}^{\text {pot }}$, that depends on the "position", i.e. on the shape parameter $\chi$.

In order to use the above dynamical framework for calculating the fission fragment mass distribution, one may proceed as follows. Starting from well inside the fission barrier (e.g. from the ground state or the last isomeric minimum), the shape evolution is followed until well beyond the last barrier, at which point the neck tends to be steadily shrinking. When the neck radius $c$ has become so small that any further change in the asymmetry is effectively precluded, the propagation is terminated and the mass split achieved is binned. By generating a sufficiently large sample of such shape trajectories (typically $\sim 10^{4}$ ) one may then obtain a histogram of the calculated fission fragment mass distribution.

Of course, no such calculation could be done until the dissipation tensor $\gamma(\chi)$ is known. Fortunately, because of the strong dissipation, one would expect that a large degree of equilibration occurs in the course of the evolution and, consequently, that the resulting mass distribution might be less sensitive to the detailed structure of the dissipation tensor. If indeed so, one might, to a first (very rough) approximation, assume that the dissipation tensor $\gamma(\chi)$ is isotropic, because in this idealized case the Smoluchowski shape evolution may be simulated $[4,5]$ by a simple Metropolis walk [10] on the five-dimensional lattice where the potential energy is available $[8,9]$.

The result of applying the Metropolis procedure to a number of actinide cases is illustrated in Fig. 2. The charge distribution is obtained from the mass distribution by scaling, $P\left(Z_{f}\right)=P\left(A_{f}\right) A_{0} / Z_{0}$, because the charge-tomass ratio is assumed to be constant throughout the fissioning system. Astonishingly good reproduction of the experimental data is achieved. The agreement is particularly good for uranium and plutonium which display welldeveloped asymmetric yields. The thorium sequence is more challenging because the yield changes from symmetric to asymmetric as neutrons are added. While this transition is well reproduced, there are significant discrepancies right where the transition occurs, $A=224-226$. But, remarkably, the differences between the calculated distributions and the corresponding experimental data are less than the differences between two neighboring distributions. So one might say that the calculation is reliable to within a single (doubly occupied) neutron orbital.
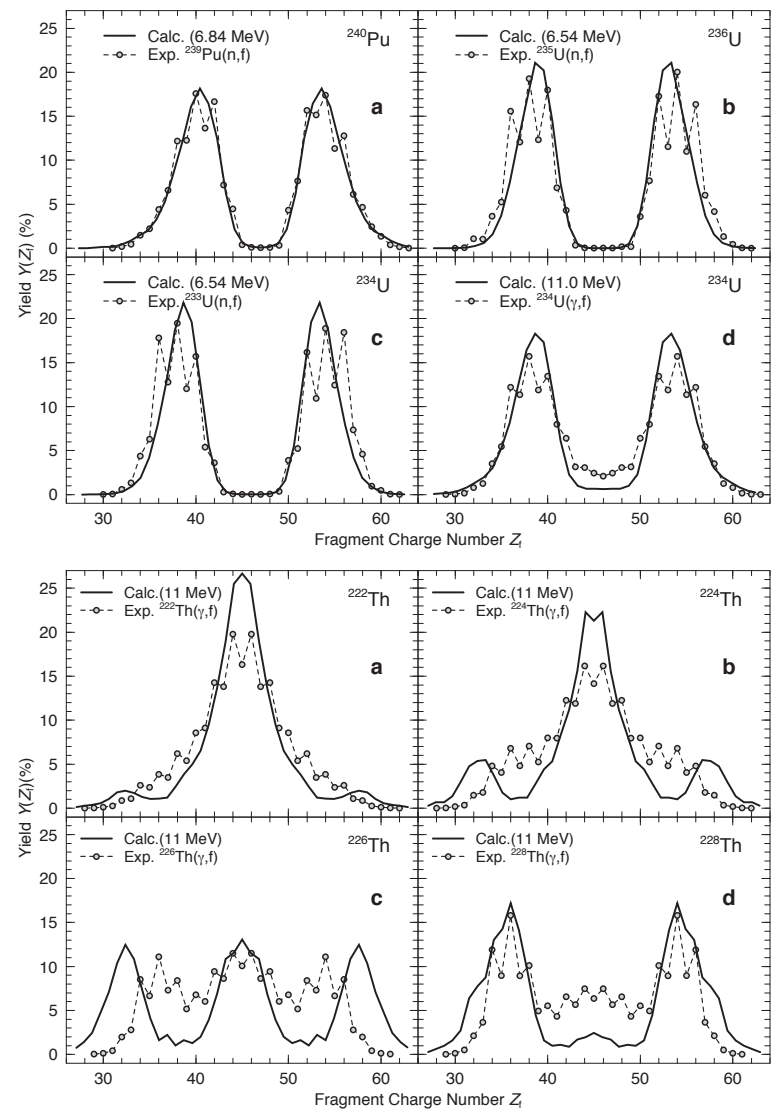

Figure 2. Fragment charge distributions extracted from Metropolis walks on the respective 5D potential-energy surfaces $[8,9]$, together with the corresponding experimental data [11, 12]. (From Ref. [4].) 


\subsection{Sensitivity to the critical neck radius}

The only parameter in the calculation of the mass distribution is the size of the neck radius at which the splits are extracted, $c_{0}$. We argued above that when $c$ is sufficiently small there is essentially no further evolution of the asymmetry. We demonstrate here that this is indeed the case.

Figure 3 shows charge distributions obtained with the simple Metropolis walk [4] using various values of $c_{0}$ (namely 2.5, 2.0, $1.5 \mathrm{fm}$ ). It is evident that the resulting distributions that are very robust against the changes in the critical neck radius $c_{0}$. Consequently, $c_{0}$ should not be considered as an adjustable parameter of the model and the method can thus be regarded as being parameter free.

The fact that there is essentially no sensitivity to precisely where the mass asymmetry is extracted suggest that it is not determined primarily by the energetics in the scission region but, presumably, rather by the broader potential-energy landscape encountered by the shape during its pre-scission evolution (see Sect. 3.2).

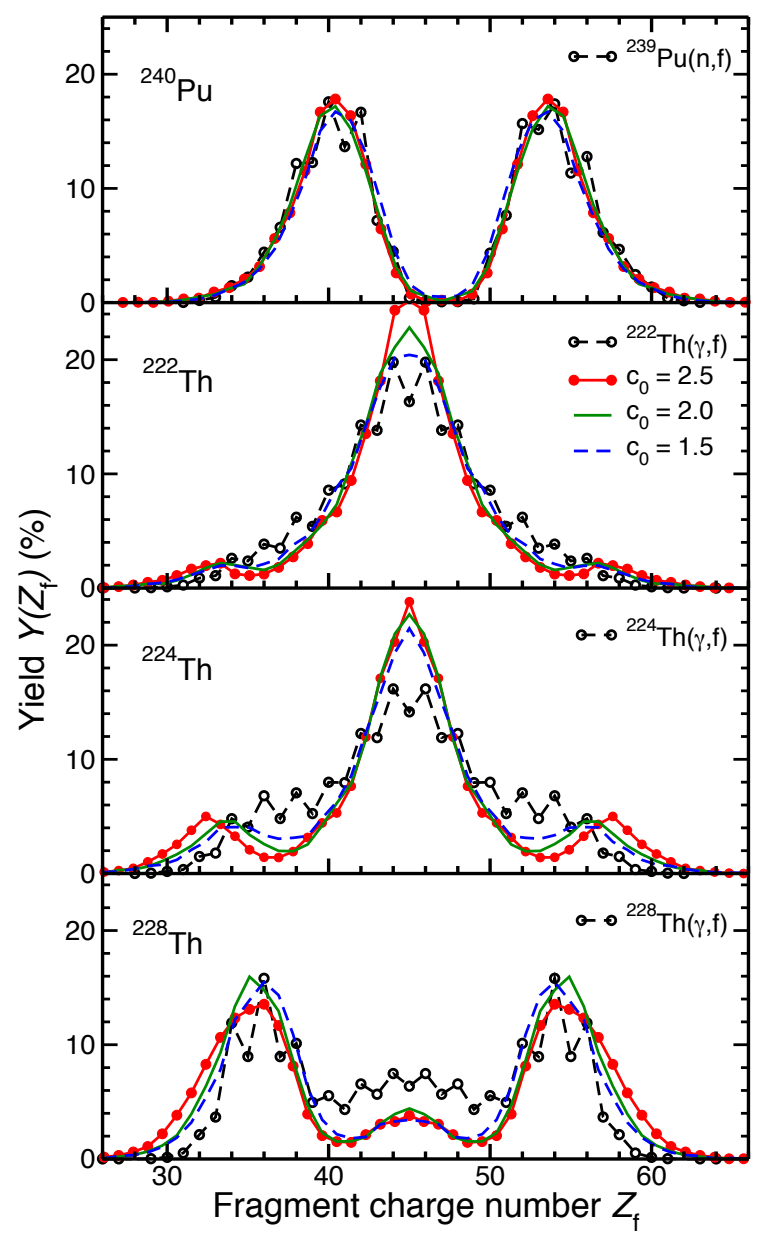

Figure 3. Charge yields for ${ }^{240} \mathrm{Pu}$ and ${ }^{222,224,228} \mathrm{Th}$ obtained with the Metropolis walk method with the mass asymmetry being extracted at various neck radii: $c_{0}=2.5,2.0,1.5 \mathrm{fm}$; the experimental data are from [11, 12]. (From Ref. [5].)

\section{Comparison with other treatments}

We discuss here briefly various investigations of the Metropolis walk method relative to other treatments.

\subsection{Shape family}

The three-quadratic-surface (3QS) shape family [6, 7] is described by five parameters (and the shapes for which the potential energy has been tabulated are labeled by $I J K L M$ ): the overall elongation (corresponding to the lattice index $I$ ); the degree of constriction (lattice index $J$ ); the reflection asymmetry determining the fragment masses (lattice index $M$ ); and the individual deformations of the two spheroidal parts $\epsilon_{\mathrm{f} 1}$ and $\epsilon_{\mathrm{f} 2}$ (indices $K$ and $L$ ) [8].

In order to illustrate the importance of employing a shape family that has a sufficient degree of flexibility, we have constructed three-dimensional potential-energy surfaces by minimizing the full five-dimensional $3 \mathrm{QS}$ surfaces with respect to $\epsilon_{\mathrm{f} 1}$ and $\epsilon_{\mathrm{f} 2}$ (corresponding to the lattice indices $K$ and $L$ ). Thus the shapes in the lowerdimensional space are characterized by only their overall elongation (represented by the lattice index $I$ ), their constriction (represented by the index $J$ ), and the degree of reflection asymmetry (represented by the index $M$ ).

Figure 4 shows the resulting charge distributions, together with the experimental data and our standard results based on the full 5D 3QS shape family. Although the 3D calculations occasionally reproduce the qualitative appearance of $P\left(Z_{\mathrm{f}}\right)$ reasonably well, the reproduction of the experimental data is generally far inferior to the results obtained with the 5D shape family. This suggests that it is of utmost importance to employ a shape family that has sufficient flexibility.
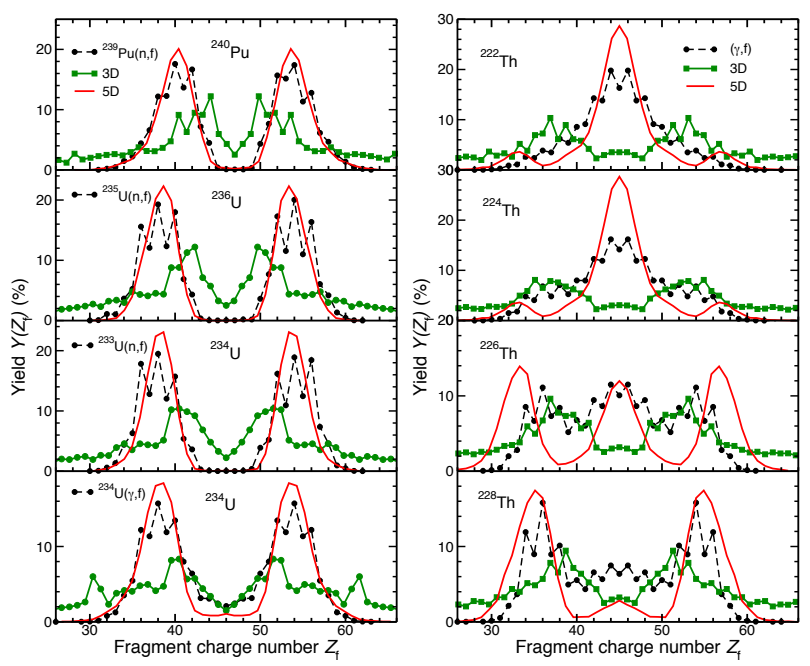

Figure 4. Charge yields resulting from Metropolis walks on the 5D potential-energy surfaces, together with the corresponding results obtained with 3D surfaces generated by minimizing the 5D surfaces with respect to the individual fragment deformations, $\epsilon_{\mathrm{f} 1}$ and $\epsilon_{\mathrm{f} 2}$; together with the corresponding experimental data [11, 12]. (From Ref. [5].) 


\subsection{Scission model}

As already recognized in the very earliest discussions of fission [2,3], the process involves a compound nucleus in which the excitation energy is distributed among the various degrees of freedom in a statistical manner. One might then imagine that statistical equilibrium is maintained all the way to scission.

However, the compound equilibrium is established only among those relatively compact shapes that are situated inside of the fission barrier, and the ensemble experiences a continual leakage as some nuclei accidentally acquire a shape far enough beyond the saddle point that they then undergo an irreversible evolution towards scission. (Elementary phase-space estimates of the leakage rate form the basis for the familiar transition-state theory of fission.) Thus the resulting fission-fragment mass distribution bears no particular relationship to the distribution of the asymmetric modes of the compound-nuclear shapes. Furthermore, because of the non-equilibrium nature of the post-barrier evolution there is no reason to expect that the resulting mass distribution should have a statistical form.

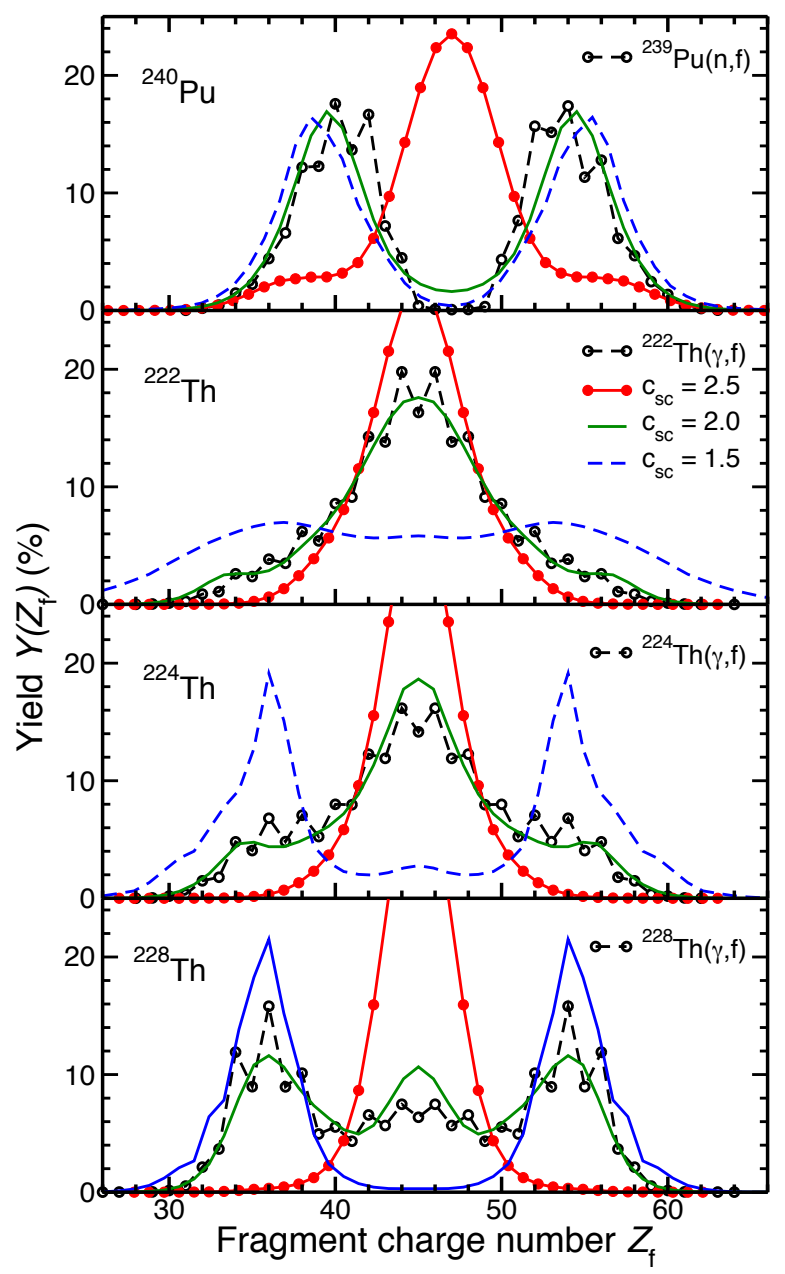

Figure 5. Results of our scission model (see text) for ${ }^{240} \mathrm{Pu}$ and ${ }^{222,224,228} \mathrm{Th}$ for scission neck radii $c_{\mathrm{sc}}=2.5,2.0,1.5 \mathrm{fm}$; the experimental data are from [11, 12]. (From Ref. [5].)
Nevertheless, a variety of statisical scission models have been studied. Indeed, models based on such considerations were among the earliest developed [13, 14] and, for each specific case, the details can often be adjusted to yield reasonable agreement with observed mass distributions. But such approaches tend to be very sensitive to the precise definition of the scission configurations adopted.

In order to illustrate this feature, we define a fourdimensional scission hypersurface as consisting of those shapes $\{\tilde{\chi}\}$ for which the neck radius equals a specified value, $c(\tilde{\chi})=c_{\mathrm{sc}}$, and we then assume that these scission configurations are populated in proportion to their statistical weight, $W(\tilde{\chi}) \sim \exp (-U(\tilde{\chi}) / T(\tilde{\chi}))$. As in the transport calculations, we use the shape-dependent temperature which ensures that the statistical weight drops rapidly to zero at the boundary of the energetically accessible region of shapes, namely those for which $U(\chi) \leq U_{\mathrm{gs}}+E^{*}$.

Results of such scission-model calculations are shown in Fig. 5 for the four representative cases also shown in Fig. 3. The character of the fragment mass distribution changes qualitatively as $c_{\mathrm{sc}}$ is decreased, whereas the Metropolis yields are robust against the changes in $c_{0}$. These results suggest that the mass split is not determined primarily by the energetics in the scission region.

\subsection{Wall dissipation}

In order to explore the dependence of the calculated mass distribution $P\left(A_{\mathrm{f}}\right)$ on the specific friction employed, we adopt the wall formula [15] which provides the one-body dissipation rate caused by the nuclear shape change,

$$
\dot{Q}_{\mathrm{wall}}=m \rho \bar{v} \int \dot{n}^{2} d \sigma=\sum_{i j} \dot{\chi}_{i} \gamma_{i j}(\chi) \dot{\chi}_{j}
$$

Because of the simple form of the wall dissipation, it is elementary to obtain the associated dissipation tensor $\gamma(\chi)$. It is generally highly anisotropic.

Because we are not interested in the time evolution but merely in the path of the shape, the overall strength of $\gamma$ is immaterial, so we renormalize it at each lattice site so its eigenvalues $\left\{\gamma_{n}\right\}$ are unity on average, $\sum_{n} \gamma_{n}(\chi)=5$. Subsequently we generate an entire family of dissipation tensors, $\tilde{\gamma}^{(f)}(\chi)$, by modifying the eigenvalues,

$$
\tilde{\gamma}_{n}^{(f)}(\chi) \equiv\left[\gamma_{n}(\chi)+f\right] /[1+f] .
$$

Then the friction tensor becomes ever more isotropic as the parameter $f$ is increased from 0 to $\infty$ and by varying $f$ through this range we may then assess the sensitivity of the mass distribution to the structure of the dissipation. (The pure wall dissipation is not fully realistic but serves well for checking the sensitivity to the dissipation tensor.)

Once the dissipation tensor $\gamma(\chi)$ has been specified, the Smoluchowski equation (4) can be solved by numerical simulation using (5). (This more elaborate propagation scheme should give the same result as the Metropolis walk if $\gamma(\chi)$ is isotropic - and does [5].) The sensitivity of the calculated charge yields to the degree of structure in the mobility tensor is illustrated in Figs. 6-7 for the eight cases shown in Fig. 2. 


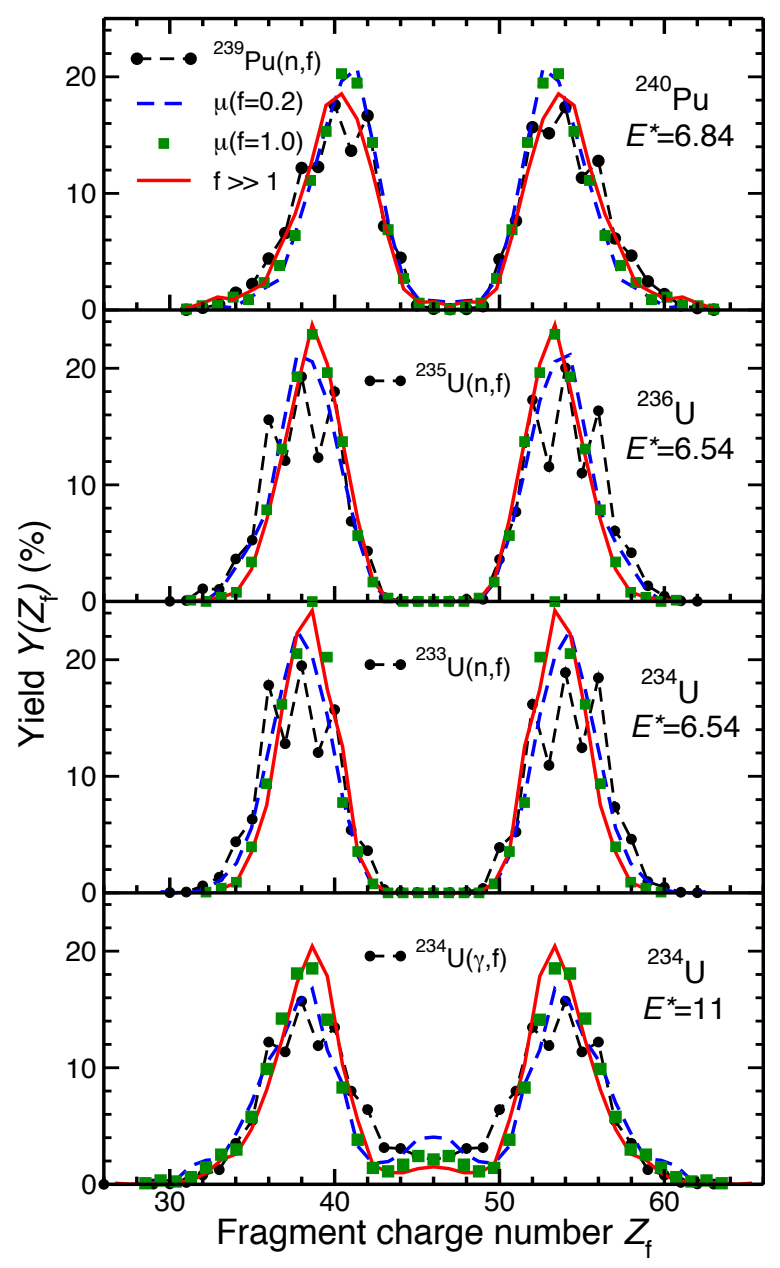

Figure 6. The fragment charge distributions for plutonium and uranium isotopes obtained by solving the Smoluchowski equation (4) for dissipation tensors having various anisotropies as specified by the parameter $f$ (see Eq. (7)), together with the experimental data [11, 12]. (From Ref. [5].)

In addition to the experimental data, each plot shows the result of three different mobility scenarios: the idealized scenario (labeled $f \gg 1$ ) where the mobility tensor is isotropic, an intermediate scenario $(f=1)$ in which the dissipation tensor is the average of the one associated with the wall formula (6) and the corresponding directional average, and a more structured dissipation $(f=0.2)$ that has an isotropic admixture of only $20 \%$.

For the plutonium and uranium cases (Fig. 6), for which the asymmetric yield is well established, there is very little sensitivity to the structure of the dissipation, but for the sequence of thorium isotopes, where the yield changes from symmetric to asymmetric as neutrons are added, the situaiton is more complicated. Generally speaking the dependence on the anisotropy parameter $f$ is remarkably small. But the central yield does exhibit a significant dependence: it grows systematically more prominent and $f$ is decreased, i.e. as the dissipation tensor acquires more structure.

Because the actual mobility tensor is not well known, it would seem prudent to employ a range of mobility sce-

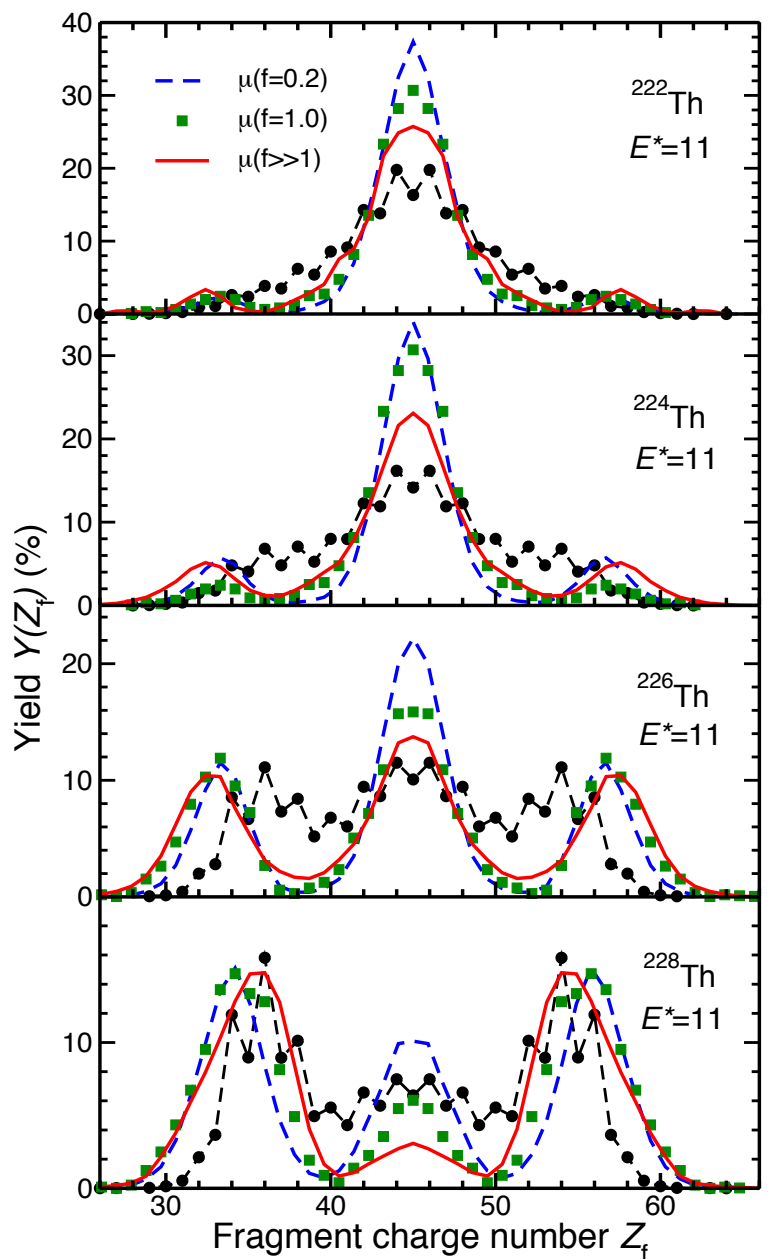

Figure 7. The fragment charge distribution for thorium isotopes obtained by solving the Smoluchowski equation (4) for dissipation tensors having various anisotropies as specified by the parameter $f$ (see Eq. (7)), together with the experimental data [11, 12]. (From Ref. [5].)

narios. The spread among the results might then be taken as a rough indication of the uncertainty in the calculation.

It should be noted that the choice of shape degrees of freedom made in Ref. [9] and of the specific 5D shape lattice used for the tabulation of the potential energy [8], was guided in large part by physical intuition (using the somewhat vague but reasonable criterion that the typical energy change between neighboring sites should be of comparable magnitude). A different choice of lattice spacing in a given direction would manifest itself in a different diffusion rate in that direction (if the density of lattice sites is increased by a certain factor then the diffusion rate is increased by the square of that factor [5]). Because the simple Metropolis walks provide mass yields that are changed only moderately when the dissipation tensor is modified, it seems that the particular choice of shape lattice was quite fortuitous. 


\section{Energy dependence}

The remarkable success of the Metropolis treatment for low-energy fission makes it natural to extend the applications towards higher excitations. For that the gradual weakening of the microscopic effects must be considered. We sketch below how it is this important feature may be incorporated by means of energy-dependent potentialenergy surfaces (for a detailed discussion, see Ref. [16]).

The potential energies employed were calculated with the macroscopic-microscopic method and thus have the form (1), $U=U_{\text {macro }}+U_{\text {micro, }}$, where both terms are available on a five-dimensional lattice of shapes. The macroscopic energy was calculated by means of the finiterange liquid-drop model $[8,9]$ and it has a smooth dependence on deformation. The corresponding macroscopic potential-energy landscape displays a single fission barrier and the associated saddle shape is reflection symmetric. By contrast, the microscopic part energy exhibits an oscillatory behavior as the shape is changed. Therefore the total potential-energy landscape has a rather bumpy appearance which for the actinides causes the ground-state shape to be deformed, generates a secondary (isomeric) minimum, and may render the outer saddle shapes asymmetric.

The tabulated potential energies $U(\chi)$ in (1) pertain to a cold nucleus, $T=0$. As the temperature is raised, both terms in (1) generally change. But the macroscopic term depends only weakly on temperature and may be assumed to remain constant. On the other hand, the microscopic term exhibits a significant temperature dependence and generally subsides as the temperature is increased; this feature may be taken into account in a simple manner by simply suppressing the term at higher excitations.

Qualitatively speaking, one expects that the microscopic term will decrease as the excitation is raised. So one may simply introduce an energy-dependent suppression of that term and define an energy-dependent effective potential[16],

$$
U_{E}(\chi) \equiv U_{\text {macro }}(\chi)+\mathcal{S}\left(E^{*}(\chi)\right) U_{\text {micro }}(\chi),
$$

where $E$ is the total energy of the fissioning system (which remains constant during the shape evolution) and $E^{*}(\chi) \equiv$ $E-U(\chi)$ is the excitation energy of the nucleus when it has the shape $\chi$. The suppression factor $\mathcal{S}\left(E^{*}\right)$ decreases from one towards zero as $E^{*}$ increases, so the usual potential $U$ is recovered for $E^{*}=0$, while only the macroscopic part remains at high excitation.

The effect of such a suppression is illustrated in Fig. 8 for a purely exponential suppression function, $\mathcal{S}\left(E^{*}\right)=\exp \left(-E^{*} / E_{0}\right)$, for various values of the damping energy $E_{0}$. The standard result is obtained for $E_{0} \rightarrow \infty$ and the yield at symmetry is not fully accounted for. But this shortfall is rectified already for a value as high as $E_{0}=60 \mathrm{MeV}$. As $E_{0}$ is lowered further, the symmetric yield increases steadily and at $E_{0}=20 \mathrm{MeV}$ the asymmetric component is hardly visible. For $E_{0}=0$ the potential is exclusively macroscopic and the charge distribution is peaked at symmetry. Because an exponential suppression with $E_{0}=60 \mathrm{MeV}$ damps the microscopic effects far too slowly, a more general suppression function is called for [16].

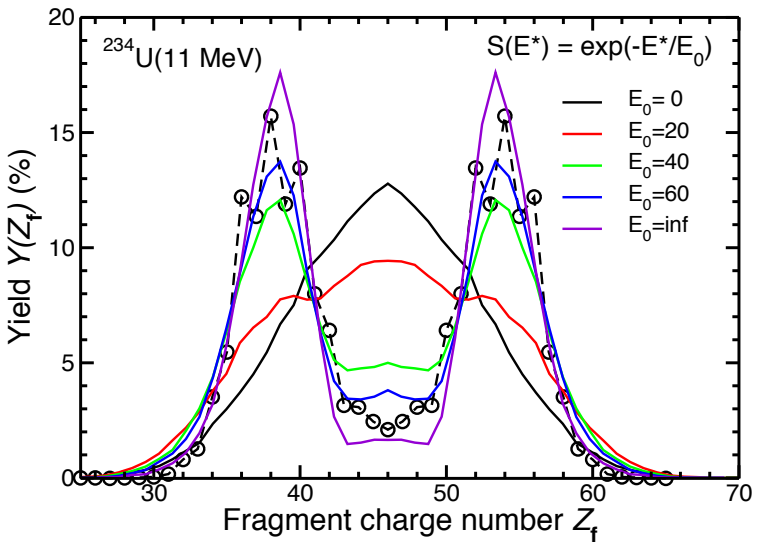

Figure 8. The relative charge yield for fission of ${ }^{234} \mathrm{U}$ at an excitation of $E^{*}=11 \mathrm{MeV}$, as calculated with a purely exponential suppression function, $\mathcal{S}\left(E^{*}\right)=\exp \left(-E^{*} / E_{0}\right)$, for various values of $E_{0}$, as indicated. The corresponding experimental data for ${ }^{234} \mathrm{U}(\gamma, \mathrm{f})$ (open circles joined by dashed lines) are from Ref. [12],

\section{Concluding remarks}

We have illustrated the utility of the simple Metropolis walk [4] on the previously calculated potential-energy lattice $[8,9]$ for obtaining the approximate form of fissionfragment mass or charge distributions for a large range of nuclei (altogether, potential-energy surfaces were calculated for more than 5,000 nuclei).

For more accurate results it is necessary to invoke also the dissipative features of the shape evolution as represented by the shape-dependent dissipation tensor. The shape evolution then resembles Brownian motion in an anisotropic (and non-uniform) medium. Because the dissipation mechanism is not yet as well understood as the potential energy, it is advisable to make a series of calculations with various forms of the dissipation tensor and then use the ensuing spread in the results as an indication of the uncertainty of the calculated mass yield. The calculation of the kinetic energies of the fission fragments is beyond the scope of the Smoluchowski treatment (and the Metropolis walk) as it would require the reintroduction of the (still poorly known) inertial-mass tensor.

The results obtained in this manner are often remarkably robust. Consequently, the method may be of practical use for calculating fission-fragment mass distributions for any of the thousands of nuclei for which the required 5D potential-energy surface is already available.

This work was supported by the Office of Nuclear Physics in the U.S. Department of Energy's Office of Science under Contract DE-AC02-05CH11231 (JR) and JUSTIPEN/UT grant DE-FG02-06ER41407 (PM), and by the National Nuclear Security Administration of the U.S. Department of Energy at Los Alamos National Laboratory under Contract No. DE-AC52-06NA25396 (PM \& AJS). 


\section{References}

[1] L. Meitner and O.R. Frisch, Nature 143, 239 (1939).

[2] N. Bohr, Nature 143, 330 (1939).

[3] N. Bohr and J.A. Wheeler, Phys. Rev. 56, 426 (1939).

[4] J. Randrup and P. Möller, Phys. Rev. Lett. 106, 132503 (2011).

[5] J. Randrup, P. Möller, and J. Sierk, Phys. Rev. C 84, 034613 (2011).

[6] J.R. Nix, Report UCRL-17958, preprint of Ref. [7].

[7] J.R. Nix, Nucl. Phys. A 130, 241 (1969).

[8] P. Möller, D.G. Madland, A.J. Sierk, and A. Iwamoto, Nature 409, 785 (2000).

[9] P. Möller et al., Phys. Rev. C 79, 064304 (2009).
[10] N. Metropolis, A.W. Rosenbluth, M.N. Rosenbluth, A.H. Teller, and E. Teller, J. Chem. Phys. 26, 1087 (1953).

[11] M.B. Chadwick et al., Nucl. Data Sheets 107, 2931 (2006).

[12] K.-H. Schmidt et al., Nucl. Phys. A 665, 221 (2000).

[13] P. Fong, Phys. Rev. 102, 434 (1956).

[14] B.D. Wilkins, E.P. Steinberg, and R.R. Chasman, Phys. Rev. C 14, 1832 (1976).

[15] J. Blocki, Y. Boneh, J.R. Nix, J. Randrup, M. Robel, A.J. Sierk, and W.J. Swiatecki, Ann. Phys. 113, 330 (1978).

[16] J. Randrup and P. Möller, Phys. Rev. C (submitted). 